\title{
Phyllodes tumors of the breast segregate in two groups according to genetic criteria
}

\author{
Marick Laé ${ }^{1}$, Anne Vincent-Salomon ${ }^{1}$, Alexia Savignoni ${ }^{2}$, Isabelle Huon ${ }^{3}$, Paul Fréneaux ${ }^{1}$, \\ Brigitte Sigal-Zafrani $^{1,4}$, Alain Aurias ${ }^{5}$, Xavier Sastre-Garau ${ }^{1}$ and Jérôme Couturier ${ }^{3}$ \\ ${ }^{1}$ Département de Biologie des Tumeurs, Service de Pathologie, Institut Curie-Hôpital, Paris, France; \\ ${ }^{2}$ Département de Biostatistiques, Institut Curie-Hôpital, Paris, France; ${ }^{3}$ Département de Biologie des \\ Tumeurs, Service de Génétique Oncologique, Institut Curie-Hôpital, Paris, France; ${ }^{4}$ Institut Curie Breast \\ Cancer Study Group, Institut Curie-Hôpital, Paris, France and ${ }^{5}$ Département de Biologie des Tumeurs, \\ Inserm U509, Institut Curie-Hôpital, Paris, France
}

\begin{abstract}
Phyllodes tumors are rare fibroepithelial tumors of the breast. The pathologic grading of phyllodes tumors based on the aspect of the stromal component, is divided into 2 or 3 grades according to the system used. To determine whether genetic markers could be of use for improving the classification of phyllodes tumors and to provide a better knowledge of the genetic alterations in these tumors, we analyzed chromosomal changes detected by comparative genomic hybridization (CGH) in comparison with histological data, in a series of 30 cases. Recurrent chromosome imbalances were observed in 55, 91 and $100 \%$ of benign, borderline and malignant phyllodes tumors, respectively. The mean number of chromosome changes was one in benign, six in borderline, and six in malignant phyllodes tumors. Most frequent genetic imbalances were $+1 q(12 / 30),-13 q$ $(7 / 30),-6 q(9 / 30),+5(9 / 30)$ and $-10 p(8 / 30)$. Gains of $1 q$, present in only one of nine benign tumors, were found in 11/21 (51\%) borderline or malignant tumors. Losses of 13q have 13q14.2 as smallest region of overlap, suggesting that the RB1 gene could be the target of deletions. Amplifications of 12q14, involving the MDM2 locus, and of 8p24, involving the MYC gene, were observed in one case each. Borderline and malignant phyllodes tumors could not be differentiated on the basis of their genomic imbalances (presence and number of chromosomal changes, presence of 1q gain and/or 13q loss). Conversely, benign tumors could be significantly differentiated from the group composed of borderline and malignant tumors $(\boldsymbol{P}<0.01)$. This study reveals two distinct patterns of genomic imbalance in phyllodes tumors: benign, with none or a few chromosome changes and malignant, with numerous recurrent chromosomal changes, in particular 1q gain and 13q loss. Helpful additional pathological criteria for differentiating the two genetic groups of phyllodes tumors are the nuclear size and the mitotic rate.

Modern Pathology (2007) 20, 435-444. doi:10.1038/modpathol.3800756; published online 2 March 2007
\end{abstract}

Keywords: phyllodes tumors; comparative genomic hybridization; FISH; MDM2; MYC; RB1

Phyllodes tumors account for less than $1 \%$ of breast tumors. ${ }^{1}$ They are fibroepithelial tumors, thought to originate from the terminal ducto-lobular unit and considered as stroma-derived. ${ }^{2}$ These biphasic tumors combine a double-layered benign epithelial component (epithelial and myoepithelial cells) and an overgrowing hypercellular mesenchymal component organized into leaf-like structures. Phyllodes tumors usually occur in the middle-aged women and usually behave in a benign fashion; however,

Correspondence: Dr M Laé, MD, Département de Biologie des Tumeurs, Service de Pathologie, Institut Curie, 26 rue d'Ulm, 75248 Paris cedex 05, France.

E-mail: marick.lae@curie.net

Received 11 August 2006; revised and accepted 04 January 2007; published online 2 March 2007 recurrences are frequent and some tumors metastasize, always as pure mesenchymal proliferations. Microscopically, the stromal component can be bland resembling that of fibroadenoma, or atypical, resembling that of soft-tissue sarcoma, or it can vary between these extremes, often resembling low-grade sarcoma. This histological variability leads to difficulties in separating phyllodes tumors in prognostically reliable categories, especially on core needle biopsies. At present, several pathological grading systems are used. Some authors separate phyllodes tumors in two subgroups, benign vs malignant, ${ }^{3,4}$ or low vs high-grade, ${ }^{5}$ whereas others classify phyllodes tumors in three categories, benign, borderline and malignant, ${ }^{1,6,7}$ or benign, low-grade malignant and high-grade malignant. ${ }^{2}$ Pathological criteria used to classify phyllodes tumors vary among 
authors. Grading is usually based on a semiquantitative evaluation of the following criteria in the stromal component: nuclear pleomorphism, mitotic rate, overgrowth, cellularity and aspects of tumor margins (infiltrating or pushing). Some authors also consider the presence of necrosis. ${ }^{6}$ In addition, the mitotic count cutoff to distinguish benign, borderline and malignant phyllodes tumors, varies according to the authors: less than $2-5$ per 10 high-power fields, and more than $3-10 .^{1,2,5,7}$ Discrepancies are also reported regarding the evaluation of stromal cellularity and atypias. Heterogeneity of phyllodes tumors between one area to another hampers this grading system. Recently, the 2003 WHO classification of tumors proposed a classification of breast phyllodes tumors in three categories (benign, borderline and malignant), with welldefined criteria. ${ }^{1}$

At present, genetic changes that characterize the course of phyllodes tumors are poorly understood. Conventional cytogenetic studies showed variable and complex changes, ${ }^{8-14}$ the significance and prognostic relevance of which remains unclear. No chromosomal aberrations specific to phyllodes tumors have been identified so far. Using comparative genomic hybridization (CGH), a technique that allows a genome-wide screening of chromosome imbalances in a single experiment, ${ }^{15,16}$ recurrent gains and losses, $+1 \mathrm{q},-3 \mathrm{p},+7 \mathrm{q},-6 \mathrm{q}$ and $-3 \mathrm{q}$, were found in phyllodes tumors. ${ }^{17}$ Other molecular studies showed that somatic or germinal mutations of TP53 were present in isolated cases of malignant phyllodes tumors. ${ }^{18,19}$ Expression of P53 was associated with histological features of malignancy, but did not predict outcome. ${ }^{20}$ The Wnt pathway $(\beta$ catenin overexpression) and the IGF pathway could play a role in the development of these neoplasms.. ${ }^{21,22}$ Increased expression of KIT has been described in phyllodes tumors, ${ }^{23-25}$ but KIT and PDGFRA activating mutations have not been identified so far. ${ }^{23,24}$ Epidermal growth factor receptor (EGFR) overexpression and amplifications are common in phyllodes tumors. ${ }^{26}$

To characterize the genetic changes associated with phyllodes tumors of the breast, we evaluated the genetic imbalances of a series of 30 phyllodes tumors of different histological grades using CGH. Our main objective was to investigate whether the genetic imbalances could help for the evaluation of the malignant potential of these tumors.

\section{Materials and methods}

\section{Tumor Samples}

Sixty-three frozen phyllodes tumors samples were available at the frozen tissue bank of Institut Curie from 1998 to 2003. A series of 30 tumors diagnosed as primary phyllodes tumors of the breast (30 patients), representing the different pathological grades (our selection does not represent the reparti- tion of phyllodes tumors in the general population) were studied. Tissue was obtained after tumorectomy or mammectomy. The pathological examination on hematoxylin and eosin (H\&E)-stained slides, carried out by at least two pathologists (ML AV-S), blindly confirmed the diagnosis and the grade using the criteria defined in the 2003 WHO classification of tumors. ${ }^{1}$ We evaluated the following criteria in the stromal component: (1) nuclear pleomorphism, (2) mitotic rate, (3) overgrowth, (4) cellularity (5) aspect of the tumor margins (infiltrating or pushing) and (6) presence of heterologous stromal elements. Stromal overgrowth was defined as an absence of epithelial component in at least one low-power field ( $\times 40$ magnification). ${ }^{6}$ The tumors were divided into benign, borderline and malignant categories. Then, a consistent, precise and quantitative pathologic evaluation was performed using cell digital image analysis (PerfectImage software, ClaraVision, Orsay, France). Stromal overgrowth was evaluated by counting the number of epithelial sections per $\mathrm{mm}^{2}$ in triplicate (the more important the stromal overgrowth is, lower is the number of epithelial sections counted). The stromal nuclear atypia were evaluated by measuring the mean nuclear surface (in $\mu \mathrm{m}^{2}$ ) of 10 nuclei on digitalized histological pictures at high-power field (hpf) of the microscope objective (objective $\times 40$, representing a total area of $0.035 \mathrm{~mm}^{2}$ ) in triplicate. The stromal cellularity was evaluated by counting the number of cells on digitalized histological pictures per hpf in triplicate. The mitotic index was evaluated per 10 consecutive neighboring fields of vision (hpf) in the most cellular areas. The microscopic aspect of tumor margins (pushing vs infiltrative) was evaluated.

\section{Tumor Processing}

Tumor tissue was frozen in liquid nitrogen immediately after surgery and stored at $-80^{\circ} \mathrm{C}$ before DNA extraction. For pathological analysis, tumor samples were fixed in acetic acid, buffered formalin, alcohol (AFA), and processed for paraffin embedding. Four micrometer sections were stained with H\&E.

\section{DNA Extraction, CGH and Image Analysis}

CGH was performed using a standard protocol, ${ }^{16}$ with minor modifications. Briefly, tumoral DNA was SpectrumGreen-labeled using a CGH nick-translation kit (Vysis, Downers Grove, IL, USA), and SpectrumRed-labeled normal male reference DNA (Vysis) was used as control DNA. For each case, $400 \mathrm{ng}$ of tumor DNA, $200 \mathrm{ng}$ of control DNA, and $50 \mu \mathrm{g}$ of Cot-1 DNA (Roche Diagnostics, Meylan, France) were mixed in $10 \mu \mathrm{l}$ of hybridization buffer and hybridized for $72 \mathrm{~h}$ at $37^{\circ} \mathrm{C}$ to a denatured normal chromosome preparation. CGH pictures from 10 metaphases were captured using an epifluorescence Leica DMRB microscope fitted with a 
Photometrics Quantix CCD camera, and analyzed with Quips software (Vysis). For DNA copy number gains, two levels of imbalances were taken into account. The first level corresponds to a green/red fluorescence ratio in the range 1.2-1.5. The second level, a ratio $\geq 1.5$, was considered as high-level amplification. For losses, a single level was taken into account, corresponding to a ratio $<0.8$.

\section{Fluorescence In Situ Hybridization}

Interphase FISH with a 12q14 band-specific digoxigenin-labeled probe encompassing $M D M 2, S A S$, GLI (Cambio, Cambridge, UK), and a SpectrumOrange-labeled $M Y C$ probe (Vysis), were performed on formalin-fixed tumor tissue section in cases 8 and 30, respectively. Each $4 \mu \mathrm{m}^{2}$ section was deparaffinized in xylene and rehydrated in a 100 85-70\% ethanol series, then washed in phosphatebuffered saline (PBS). After a proteolytic treatment using a pretreatment kit (Vysis) for formalin-fixed samples, the tissue section and probe were codenatured at $95^{\circ} \mathrm{C}$ for $5 \mathrm{~min}$, and hybridized for $15 \mathrm{~h}$ at $37^{\circ} \mathrm{C}$. Slides were washed in $2 \times \mathrm{SSC} / 0.3 \%$ Igepal at $75^{\circ} \mathrm{C}$, for $5 \mathrm{~min}$, then in PBS/Tween 20 at $20^{\circ} \mathrm{C}$. For the 12q14 probe, detection was achieved using a rhodamine-coupled anti-digoxigenin antibody (Roche Diagnostics), diluted 1/50. Nuclei were counterstained using a DAPI/antifade solution. Slides were viewed with a double-band DAPI/ rhodamine filter, and images were captured as described for CGH. At least 50 interphase nuclei were analyzed per hybridization.

\section{Statistical Analysis}

The qualitative variables are presented in terms of percent and the quantitative in terms of means, standard error, median, minimum and maximum.
Comparisons between pathological and genetic data were performed by using exact Fisher test in the relevant contingency tables in case of qualitative variables, or Kruskal-Wallis test in case of quantitative variables. To evaluate the independent influence of stromal size, mitotic index and stromal cellularity on the group discrimination, a multivariate analysis was performed using a logistic regression. A predictive score was proposed to discriminate the group composed of benign tumors to the one composed of borderline and malignant ones. It was calculated as an addition of the factors selected by the multivariate analysis, respectively multiplied by their regression coefficients. To categorize this score, the mean of the quantitative variable were chosen. This sensibility and specificity were given associated with their respective 95\% confidence interval.

Two-sided $P$-values below 0.05 were considered significant. The analysis was realized using S-Plus 2000 software (MathSoft Inc., Seattle, WA, USA).

\section{Results}

\section{Pathologic Features}

Phyllodes tumors were classified in a three-tierce system as described above. Among the 30 cases, 9 were classified as benign, 12 as borderline and 9 as malignant phyllodes tumors. The pathologic features are detailed in Table 1. The median value of the number of epithelial sections per $\mathrm{mm}^{2}$ representing the stromal overgrowth, was statistically different between the three groups: 11 (range 6-30) for benign, 5.6 (2-15) for borderline, and 3 (1-17) for malignant phyllodes tumors. The median nuclear size of the mesenchymal tumor cells at high-power field was $41 \mu \mathrm{m}^{2}$ (range 35-53) for benign, $58 \mu \mathrm{m}^{2}$ (42-146) for borderline and $85 \mu \mathrm{m}^{2}$ (55-173) for malignant PT $(P<0.001)$. The median number of

Table 1 Clinicopathological data of the 30 phyllodes tumors studied

\begin{tabular}{|c|c|c|c|c|}
\hline & $\begin{array}{c}\text { Benign }(\mathrm{n}=9)(\text { median } \\
(\text { min-max }))\end{array}$ & $\begin{array}{l}\text { Borderline }(\mathrm{n}=12) \\
(\text { median }(\min -\max ))\end{array}$ & $\begin{array}{c}\text { Malignant }(\mathrm{n}=9) \\
(\text { median }([\min -\max ))\end{array}$ & $\mathrm{P}$-value \\
\hline Age (years) & $36(22-50)$ & $46(22-74)$ & $54(47-64)$ & 0.007 \\
\hline Tumor size (mm) & $40(20-70)$ & $45(10-200)$ & $60(20-250)$ & 0.4 \\
\hline Stromal cellularity ${ }^{\mathrm{a}}$ & $85(52-147)$ & $152(53-250)$ & $210(95-358)$ & $<10^{-3}$ \\
\hline Stromal overgrowth ${ }^{\mathrm{b}}$ & $11(6-30)$ & $5.6(2-15)$ & $3(1-17)$ & 0.023 \\
\hline Nuclei size $\left(\mu \mathrm{m}^{2}\right)$ & $41(35-53)$ & $58(42-146)$ & $85(55-173)$ & $<10^{-3}$ \\
\hline Mitotic rate ${ }^{\mathrm{c}}$ & $1(0-9)$ & $5.5(2-17)$ & $18(5-50)$ & $<10^{-3}$ \\
\hline
\end{tabular}

Abbreviations: B, benign; BL, borderline; I, infiltrative; M, malignant; P, pushing.

${ }^{\mathrm{a}}$ Number of stromal cells per HPF.

${ }^{\mathrm{b}}$ Number of epithelial sections per $\mathrm{mm}^{2}$.

${ }^{\mathrm{c}}$ Number of mitotic figures per $10 \mathrm{HPF}$. 
stromal cells per high-power field was 85 (range 52147) for benign, 152 (range 53-250) for borderline and 210 (range 95-358) for malignant tumors $(P<0.001)$. The median mitotic index per $10 \mathrm{hpf}$ was one (range 0-9), $5.5(2-17)$ and $18(5-50)$ for benign, borderline and malignant tumors, respectively $(P<0.001)$. Microscopically, all nine benign tumors had pushing margins, whereas 6/12 (50\%) borderline and $7 / 9(78 \%)$ malignant tumors had infiltrative margins $(P=0.002)$. The stromal component represented over $80 \%$ of the total cells for each tumor sample, indicating that CGH results reflect genomic balance of the stromal component. The microscopic appearance of one tumor (case 19) is illustrated in Figure 3c.

\section{Clinical Features}

Clinical features are summarized in Table 1. The median age at diagnosis was 36-year-old (range 22$50)$ for benign, $46(22-74)$ for borderline and 54 (47$64)$ for malignant phyllodes tumors $(P=0.007)$. The median tumor size was $40 \mathrm{~mm}$ (range 20-70), $45 \mathrm{~mm}$ (10-200), $60 \mathrm{~mm}(20-250)$ in benign, borderline and malignant tumors, respectively (no statistical differences).

\section{CGH Features}

CGH results of the 30 phyllodes tumors are summarized in Tables 2 and 3. A map of chromosome imbalances detected is shown in Figure 1. Recurrent chromosome imbalances were identified in $83 \%(25 / 30)$ of the tumors and in $55 \%, 91 \%$ and $100 \%$ of benign, borderline and malignant phyllodes tumors, respectively $(P=0.045)$. The most frequent gains involved chromosome arm 1q (12/ $30)$, and chromosomes 5 (9/30), and 18 (5/30). Loss of chromosomal material was most frequently found at $13 q(7 / 30), 6 q(9 / 30), 10 p(8 / 30), 12 q(6 / 30)$. The smallest region of overlap (SRO) of $13 \mathrm{q}$ is the 13q14.2 region. A CGH profile of a malignant phyllode tumor (case 22) is shown in Figure 2a.
High-level gains (fluorescence ratio $\geq 1.5$ ), suggesting the existence of amplifications, were observed in two cases: in bands 7p11 and 12q14 (case 28), and in band 8q24 (case 30) (Figure 2b). Interphase FISH with specific 12q14 probe encompassing MDM2, SAS and GLI showed clusters of signals in cells of the stromal component only, suggesting the presence of hsr(s) representing 8-10 copies of this band (Figure 3a). The epithelial component did not show amplification. Amplification of MYC (6 copies), located in 8q24, was also confirmed by FISH in case 30 in the stromal component (Figure 3b).

When comparing the number of chromosome imbalances to the type of tumors, we observed that there was a significant statistical difference between the three groups. Almost half of benign tumors $(44 \%)$ did not show any chromosomal changes. In contrast, $91 \%$ of borderline (11 cases) and $100 \%$ of malignant phyllodes tumors (9 cases) presented chromosomal imbalances $(P=0.045)$ (Table 2). Benign tumors showed a median of one chromosomal change (range 0-3), mainly loss of a whole chromosome 6 , borderline tumors a median of six changes (range: 0-13), and malignant phyllodes tumors, six changes (range: 1-20) $(P=0.003)$ (Table 2). Borderline and malignant phyllodes tumors could not be differentiated on the basis of the number of chromosomal imbalances $(P=0.83)$. In contrast, benign tumors could be differentiated from the group composed of borderline and malignant tumors based on the number of chromosomal changes $(P<0.01)$.

Gain of $1 \mathrm{q}$ and loss of $13 \mathrm{q}$ were among the most common chromosomal changes in phyllodes tumors, present in 40 and $23 \%$ of cases, respectively. At least one of these changes was present in $53 \%$ of phyllodes tumors. Furthermore, one or more of the four most frequent chromosomal changes were present in 19/21 (90\%) of borderline and malignant tumors. We found a significant difference between the three pathological categories in term of presence of $1 \mathrm{q}$ gain and/or $13 \mathrm{q}$ loss $(P=0.01)$ : $1 / 9(11 \%)$ benign tumors, $9 / 12(75 \%)$ borderline tumors and $6 /$ $9(66 \%)$ malignant phyllodes tumors presented 1q

Table 2 Chromosome imbalances detected by CGH in 30 phyllodes tumors

\begin{tabular}{|c|c|c|c|c|}
\hline & $\begin{array}{l}\text { Benign }(\mathrm{n}=9) \\
\text { No } \text { cases }(\%)\end{array}$ & $\begin{array}{l}\text { Borderline }(\mathrm{n}=12) \\
\quad \text { No cases }(\%)\end{array}$ & $\begin{array}{l}\text { Malignant }(\mathrm{n}=9) \\
\text { No cases }(\%)\end{array}$ & $\mathrm{P}$-value \\
\hline Cases with chromosome imbalances & $5(55)$ & $11(91)$ & $9(100)$ & 0.045 \\
\hline 1q gain & $1(11)$ & $7(58)$ & $4(44)$ & 0.096 \\
\hline $13 q$ loss & 0 & $4(33)$ & $3(33)$ & 0.15 \\
\hline Tumors with $1 \mathrm{q}$ gain and/or $13 \mathrm{q}$ loss & $1(11)$ & $9(75)$ & $6(66)$ & 0.01 \\
\hline Mean number of imbalances (range) & $1(1-3)$ & $6(0-13)$ & $4(1-20)$ & 0.003 \\
\hline
\end{tabular}

Abbreviation: CGH, comparative genomic hybridization. 
Table 3 Distribution of recurrent chromosomal imbalances in 30 phyllodes tumors

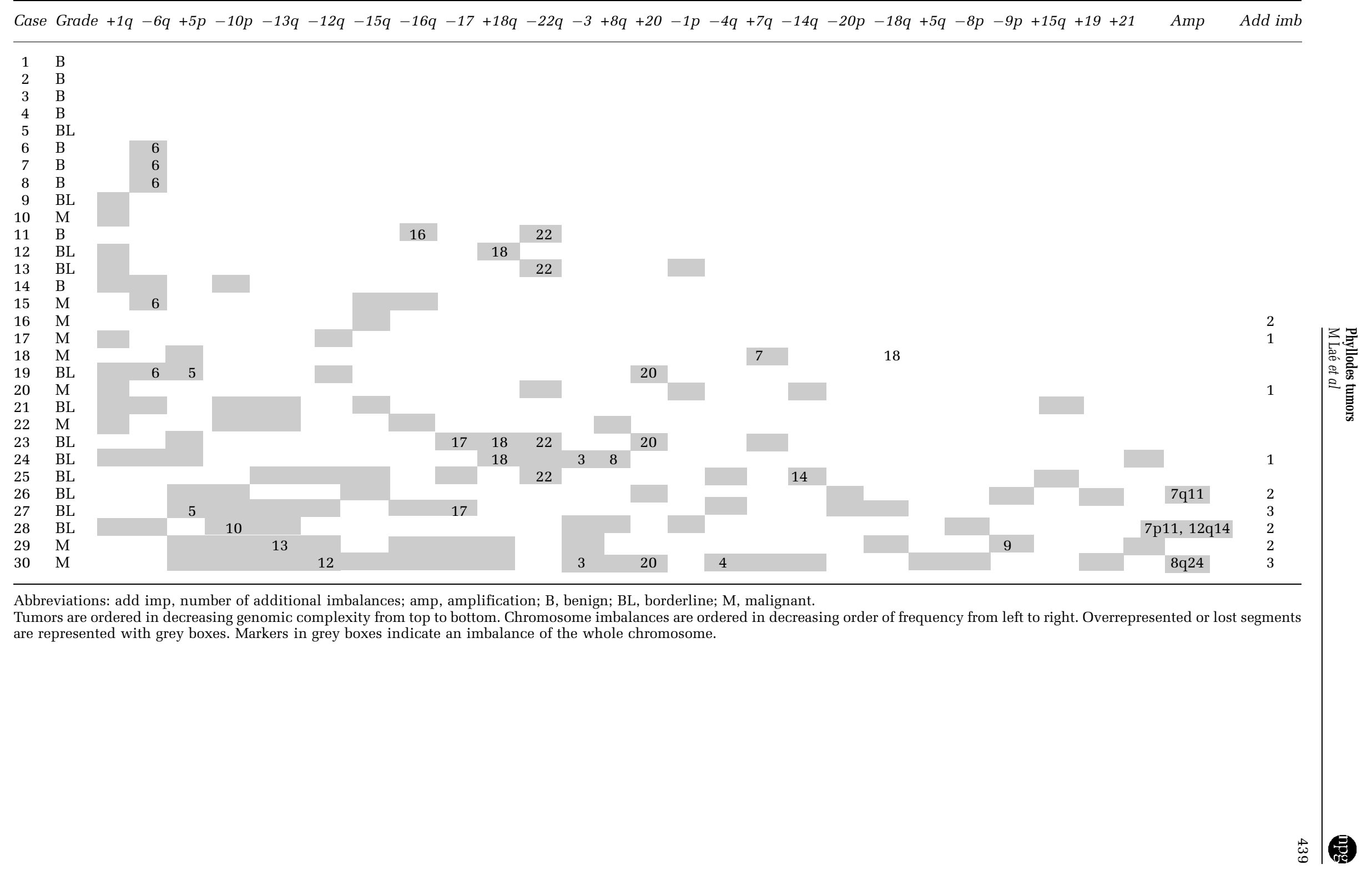



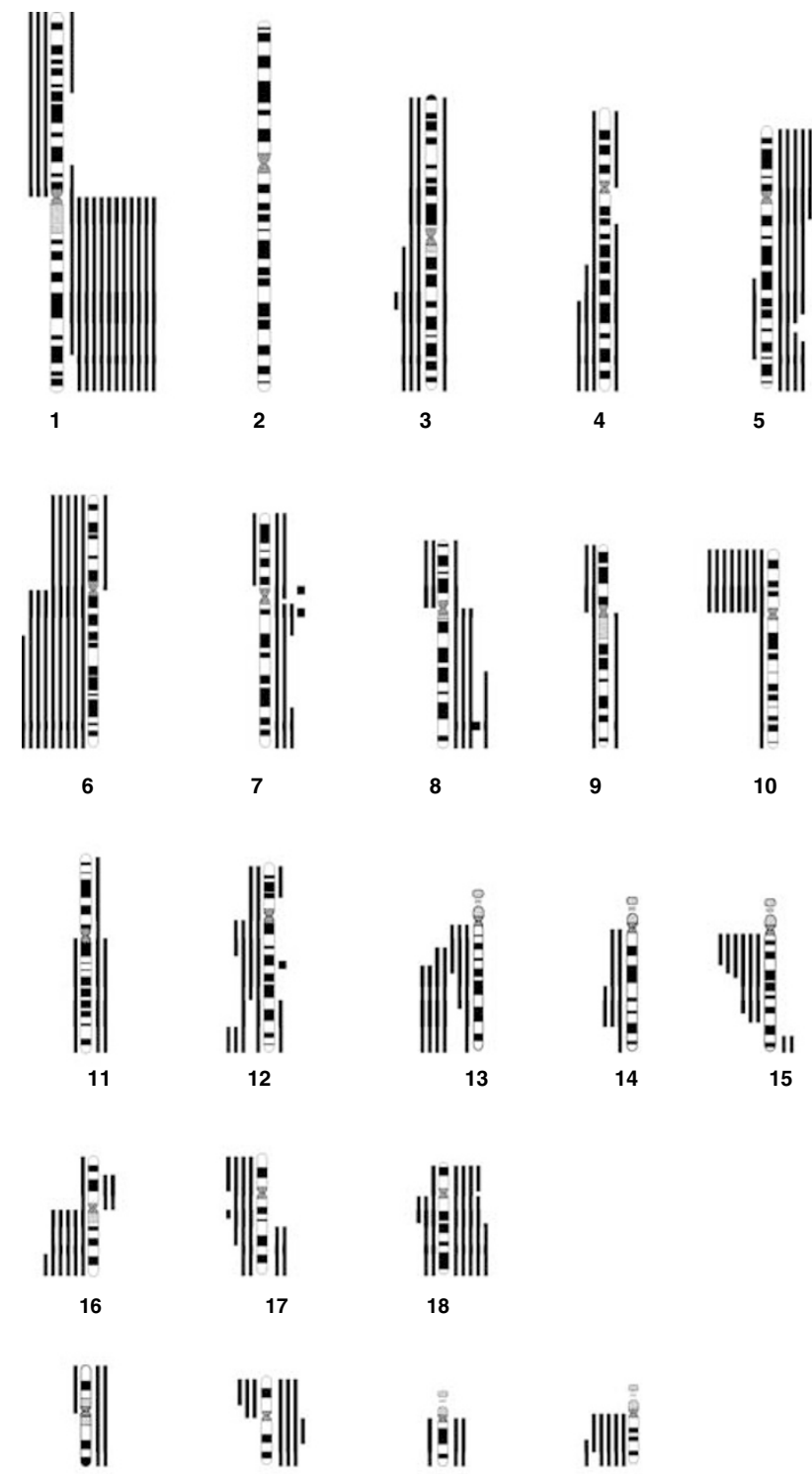

19

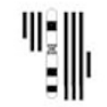

20

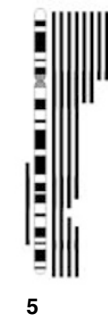

Figure 1 Ideogramm representing a summary CGH results of 30 phyllodes tumors. Each line represents a single tumor. Five additional tumors did not show any alterations. Chromosomal region gains are represented by vertical lines on the right side of the chromosomes and losses by vertical lines on the left side. Black squares on chromosomes 7,8 and 12 correspond to highlevel amplifications.

gain and/or $13 \mathrm{q}$ loss. There were no significant differences between borderline and malignant phyllodes tumors. In contrast, there was a significant difference when comparing the benign phyllodes tumors from the group composed of borderline and malignant phyllodes tumors $(P=0.005)$ : 15/21 (71\%) borderline and malignant phyllodes tumors and $1 / 9(11 \%)$ of benign phyllodes tumors presented 1q gain and/or 13q loss. As two group of phyllodes tumors could be identified (the group of benign tumors and the group of borderline/malignant ones) according to the genetic data, we focused our study on the pathological criteria that could help to separate them. Univariate analysis identified two significant pathological criteria, namely, nuclear size and mitotic activity. Other criteria were nonsignificant. Multivariate analysis of CGH criteria (absence or presence of chromosomal changes, number of chromosomal changes, presence of $1 \mathrm{q}$ gain and/or 13q loss) showed that only the presence of $1 \mathrm{q}$ gain and/or $13 \mathrm{q}$ loss had an independent predictive value to separate the two categories (benign vs borderline and malignant). Another multivariate analysis using the pathological criteria significant in univariate analysis and the presence of $1 \mathrm{q}$ gain and/or $13 \mathrm{q}$ loss, indicated that the presence of $1 \mathrm{q}$ gain and/or $13 \mathrm{q}$ loss has no independent predictive value. Therefore, only the pathological criteria were taken into account to predict the belonging to one of the two groups (the group of benign tumors and the group of borderline/ malignant ones). The score took into account the pathological criteria that were independent predictive factors in multivariate analysis (namely, mitotic activity and nuclear size, $P=0.04$ and $P<0.001$, respectively). All phyllodes tumors presenting none or a few chromosome changes are characterized by a nuclear size $<50 \mu \mathrm{m}^{3}$ and/or a mitotic rate $<3 / 10 \mathrm{HPF}$ and/or a cellularity $<100$ nuclei/1 HPF. All phyllodes tumors presenting numerous recurrent chromosomal changes were characterized by a nuclear size $\geq 50 \mu \mathrm{m}^{3}$ and/or a mitotic rate $\geq 3 / 10 \mathrm{HPF}$ and/or a cellularity $\geq 100$ nuclei/ $1 \mathrm{HPF}$. A predictive score was then calculated from the coefficient found by this model multiplied by the pathological criteria (score $=2.90$ mitotic index +3.5 nuclear size). The mean score was 4.2 (range 0-6.4), with a standard error equal to 2.8. We decided to use a cutoff given a class of score $\leq 4$ and $\geq 4$. A score $\leq 4$ classifies the tumor as benign and a score $\geq 4$ as borderline/malignant. This score has been simplified: a tumor presenting none or only one criteria (nuclear size $\geq 50 \mu \mathrm{m}^{3}$ or a mitotic rate $\geq 3 / 10 \mathrm{HPF}$ ) was classified as benign and a tumor presenting two criteria was classified as borderline/malignant. We found that the sensibility of this score was $81 \%(58-94 \%)$ and its specificity $100 \%(67-100 \%)$. Three cases were misclassified (all of them were initially diagnosed as borderline tumors).

\section{Discussion}

The present study represents a genome-wide investigation of the genetic imbalances of a series of 30 phyllodes tumors of the breast, in relation to the pathologic grading. It reveals that $25 / 30(83 \%)$ of the samples showed chromosomal imbalances. These alterations involved at least one or more often several chromosomal regions. Our results pinpoint several chromosomal regions where gains, losses or amplifications were noted. The most recurrent of 

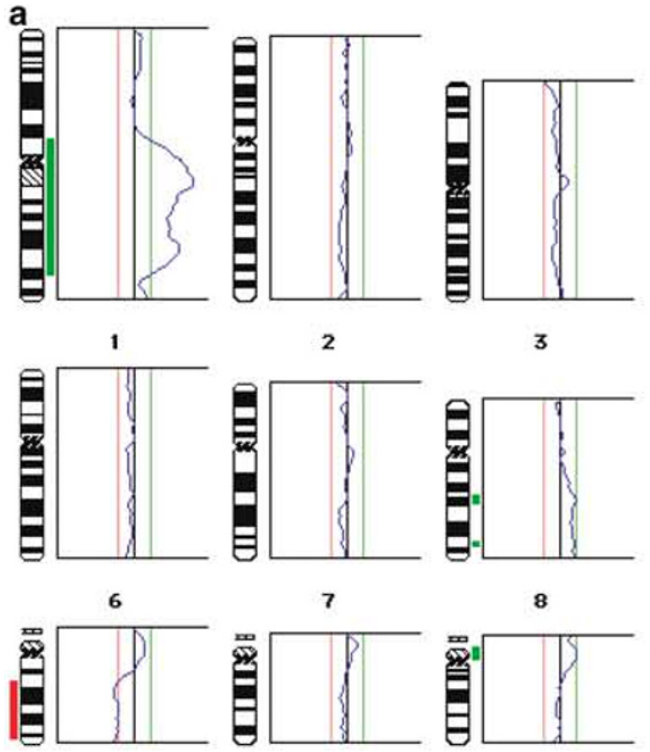

13

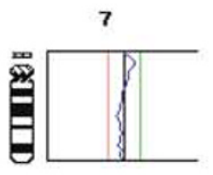

14

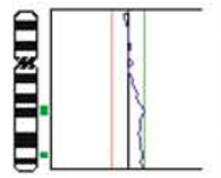

8
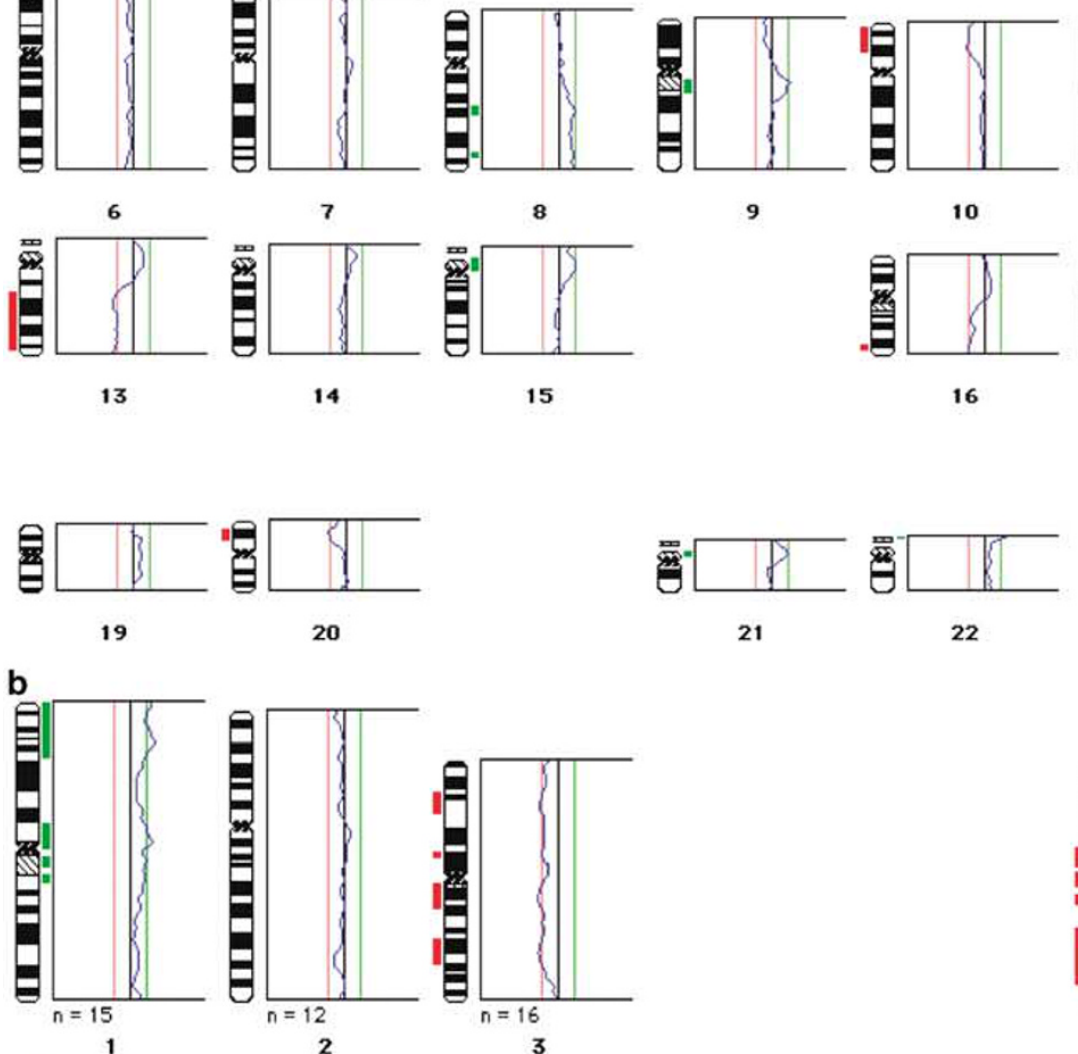

9
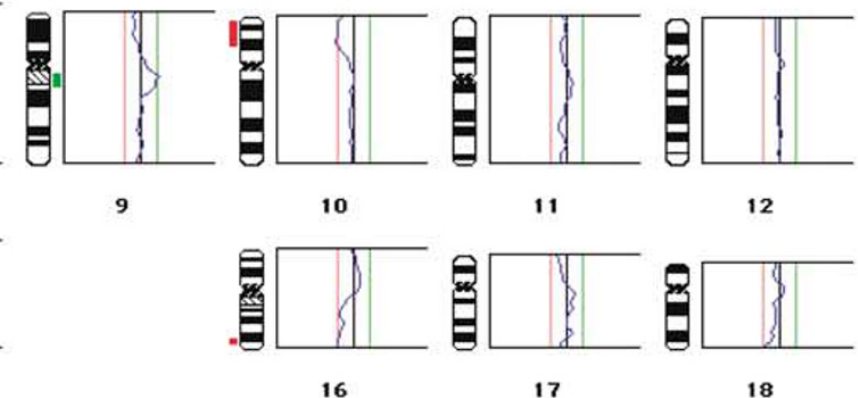

11
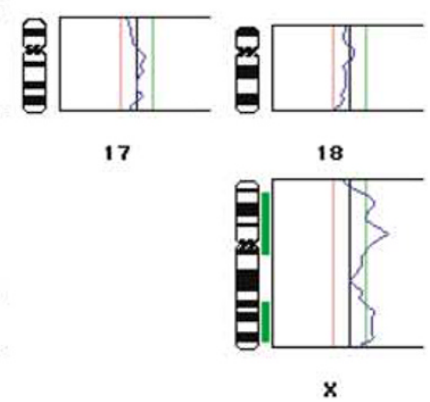

$x$
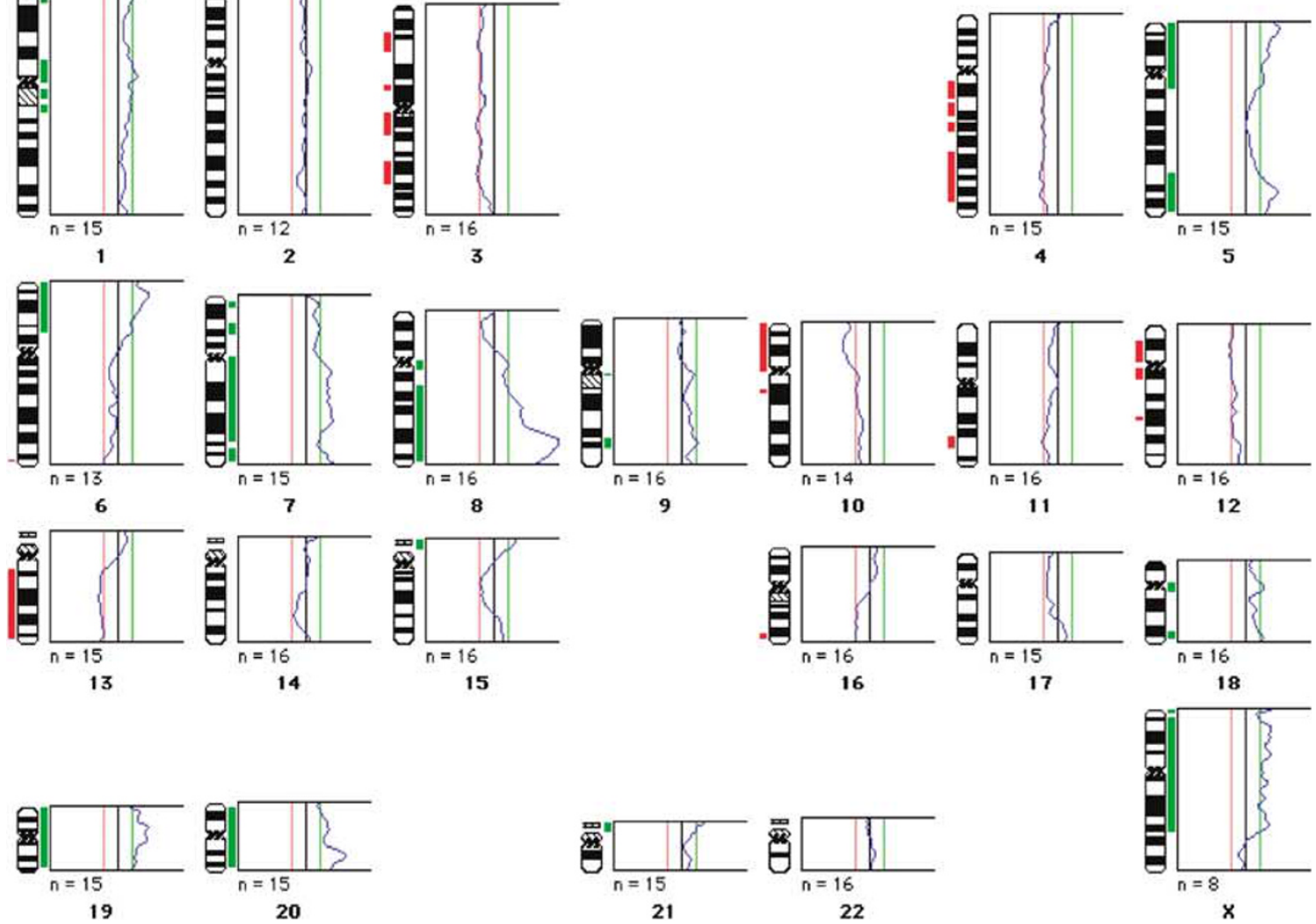

Figure 2 CGH profiles of two characteristic cases. (a) Case 22. Notice 1q gain and 13q loss, among other imbalances. (b) Case 30. Notice high-level amplification centered on band 8q24. 

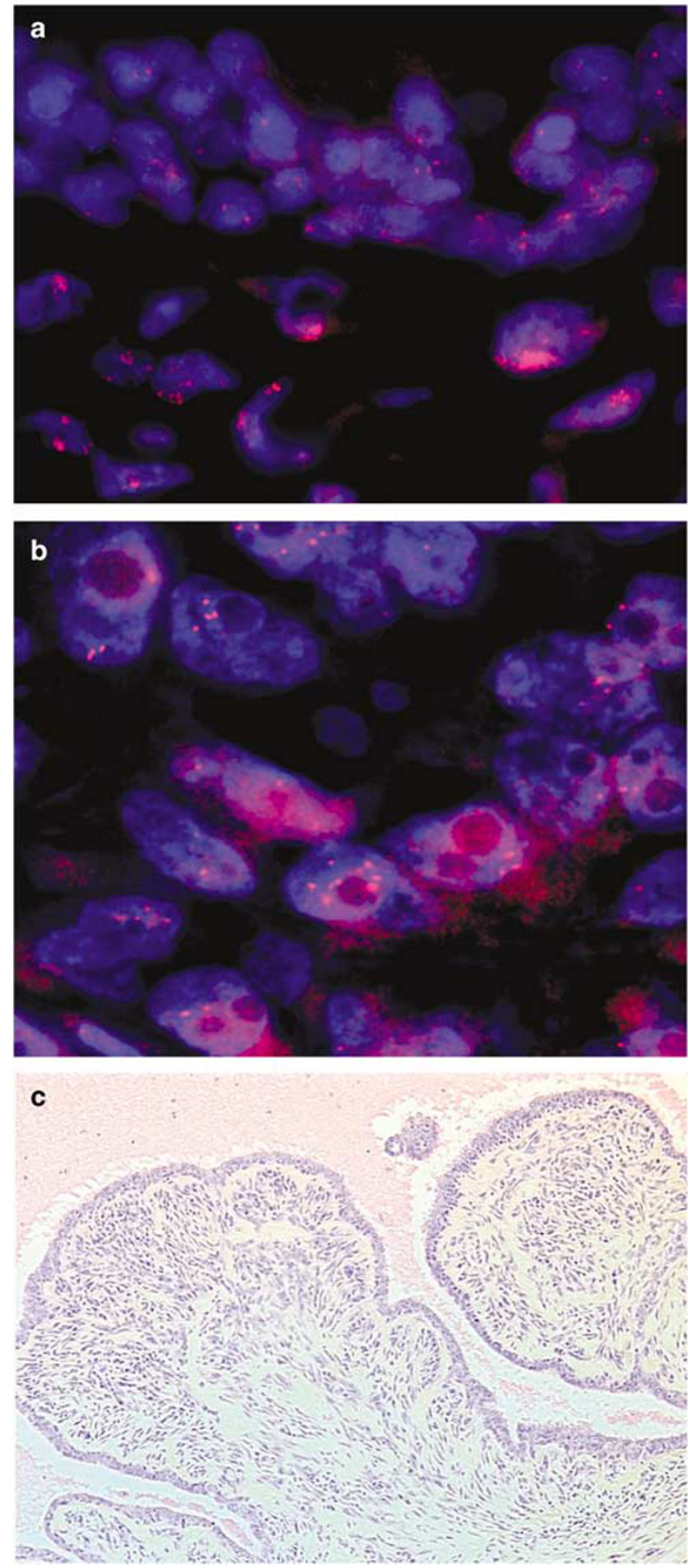

Figure 3 Interphase FISH on paraffined-embedded tumor tissue section of two cases showing amplification. (a) Case 19: FISH with a rhodamine-labeled 12q14 (encompassing MDM2) probe showing clusters of 8-10 signals in the stromal component. Notice that epithelial cells do not show amplification. (b) Case 23: FISH with a rhodamine-labeled $M Y C$ probe on formalin-fixed, paraffined-embedded tumor tissue section showing a mean of 6 signals. (c) H\&E tissue section of case 19 (borderline phyllode tumor). them probably contains genes implicated in the genesis of phyllodes tumors. Gains of $1 \mathrm{q}$ and $13 \mathrm{q}$ losses have emerged as the hallmark alterations in phyllodes tumors of the breast.

The most frequent alteration was gain of $1 \mathrm{q}$, present in $40 \%$ of the tumors $(12 / 30)$. With the exception of one benign case (Case 14), 1q gain was found only in borderline and malignant categories. Gain of $1 \mathrm{q}$ is may be a secondary change providing a selective growth advantage during tumor progression. It generally corresponds to an unbalanced translocation of $1 \mathrm{q}$ arm with various partner chromosomes or to an isochromosome $1 \mathrm{q}$, or a $1 \mathrm{q}$ duplication. Gain of $1 \mathrm{q}$ is one of the commonest changes observed in both pediatric and adult malignant solid tumors. ${ }^{27}$ It is present in over $50 \%$ of a wide range of malignant human tumors ${ }^{28-30}$ such as breast carcinomas, ${ }^{28,31-33}$ esophageal squamous cell carcinomas, ${ }^{34}$ retinoblastomas, ${ }^{35,36}$ neuroblastomas ${ }^{37}$ and leukemias among others.

Loss of $13 \mathrm{q}$ was present in 7 of 30 phyllodes tumors $(23 \%)$. It was observed only in the borderline (4/12) and malignant (3/9) cases. Loss of $13 q$ has been described in several types of solid tumors, ${ }^{38}$ including epithelial, mesenchymal and neuronal neoplasms as well as in hematopoeitic neoplasms. ${ }^{39}$ Over $30 \%$ of sarcomas, for example, 'so called' malignant histocytofibroma, ${ }^{40-42}$ liposarcomas ${ }^{41}$ or leiomyosarcoma ${ }^{43}$ present $13 q$ loss. Furthermore, loss of $13 \mathrm{q}$ has $13 \mathrm{q} 14.2$ as SRO in the 7 tumors, suggesting that a gene localized in these regions could act as a tumor suppressor gene and that its inactivation could be relevant in phyllodes tumors oncogenesis. The $R B 1$ gene localized in this region could be the target of deletions. The putative role of $R B 1$ in phyllodes tumors oncogenesis and/or progression should be evaluated by further studies.

Chromosome gain of $1 \mathrm{q}$ and $13 \mathrm{q}$ are significantly associated with borderline and malignant tumors. Overall, these results suggest that analyses using pangenomic techniques such as CGH or array-CGH could be helpful in grading phyllodes tumors for which a precise pathological grade is difficult to evaluate solely on H\&E slides.

Genomic amplification is clearly associated with tumor progression in human cancer and occurs lately during tumorigenesis. ${ }^{44}$ Genomic amplification seems to play a role in phyllodes tumors pathogenesis as MDM2 and MYC were amplified in one phyllode tumor each. $M Y C$ is amplified in a broad range of epithelial tumors, among them breast carcinoma. ${ }^{45}$ Interestingly, a case of phyllodes tumors with high-level amplification of $M Y C$ in the stromal component has also been referred. ${ }^{24}$

$M D M 2$, a negative regulator of p53 was amplified in one of our phyllodes tumors cases. MDM2 is found to be frequently amplified in some sarcomas such as osteosarcomas, ${ }^{46}$ liposarcomas, ${ }^{47,48}$ 'socalled' malignant histocytofibromas, ${ }^{39,43}$ leiomyosarcomas $^{43}$ and neuroglial tumors. ${ }^{49}$ The fact that in our 
series of tumors, phyllodes tumors are characterized by frequent loss of $13 q$ and occasional amplification of MDM2 further connect malignant phyllodes tumors to sarcomas.

This study has provided evidence that borderline and malignant phyllodes tumors could not be differentiated on the basis of the presence and of the number of chromosomal imbalances. This suggests that only two types of phyllodes tumors could be distinguished on a genomic basis: benign phyllodes tumors and malignant phyllodes tumors (which includes the borderline and malignant pathologic categories). We showed that a precise pathological evaluation of the two pathological criteria (nuclear size and mitotic index) is a helpful adjunct to differentiate the two genetic groups of phyllodes tumors. Our statistical score presented a large range in the assessment of the sensibility and specificity given the limited number of tumors analyzed. An evaluation of this score in a large number of phyllodes tumors is needed for validation. It would be of interest to perform CGH on an external set of phyllodes tumors to evaluate if the benign and malignant genetic categories correlate with the pathological categories. As follow-up was available for 14 patients only (15-48 months), we could not draw any conclusions regarding recurrence and metastatic rate.

This study further defines the genetic alterations of phyllodes tumors. Our results are in concordance with data from the literature showing alterations in $79 \%$ of a series of 18 phyllodes tumors, and demonstrating $1 \mathrm{q}$ gain in $38 \%{ }^{17}$ and $55 \%$ of phyllodes tumors. ${ }^{50}$ In contrast to our results, Lu et $a l^{17}$ mainly found $1 \mathrm{q}$ gain in benign tumors $(6 / 15$ benign phyllodes tumors). This discrepancy highlights the difficulties in grading phyllodes tumors. It is emphasized that in this later report, the grading did not take into account the stromal cellularity and did not quantify precisely the stromal overgrowth or the nuclear size. In summary, when pooling our data with Lu et $a l,{ }^{17}$ phyllodes tumors are characterized by the following recurrent chromosomal changes: $+1 \mathrm{q}(42 \%),-6 \mathrm{q}(27 \%),-13 \mathrm{q}(20 \%),-10 \mathrm{p}(22 \%)$, $+5 \mathrm{p}(22 \%)$.

In conclusion, this study brings new insights in the pattern of genetic alterations that may be associated with the development and progression of phyllodes tumors. It illustrates that combined CGH and FISH analysis is a valuable approach to identify genes associated with the progression of these tumors with complex genomic alterations. The CGH results show that a classification of phyllodes tumors of the breast into two categories, benign and malignant, better reflects the genetic reality. Further studies are required to validate the importance of these genomic alterations. Studies of chromosomal regions highlighted by CGH using array-CGH would be of particular interest in order to identify oncogenes or tumor suppressor genes that could be activated or inactivated in phyllodes tumors.

\section{References}

1 Tavassoli FA, Devilee P (eds). World Health Organization Classification of Tumors. Pathology \& Genetics of Tumors of the Breast and Female Genital Organs. International Agency for research on Cancer Press: Lyon, 2003, pp 99-103.

2 Rosen PP. Cystosarcoma phyllodes. Fibroepithelial neoplasms. In: Rosen PP (ed). Rosen's Breast Pathology. Lipincott-Raven: PA, 2001, pp 143-175.

3 Layfield LJ, Hart J, Neuwirth H, et al. Relation between DNA ploidy and the clinical behavior of phyllodes tumors. Cancer 1989;64:1486-1489.

4 McDivitt RW, Urban JA, Farrow JH. Cystosarcoma phyllodes. Johns Hopkins Med J 1967;120:33-45.

5 Tavassoli FA. Pathology of the Breast, 4 edn. McGrawHill: New York, 1999.

6 Moffat CJ, Pinder SE, Dixon AR, et al. Phyllodes tumours of the breast: a clinicopathological review of thirty-two cases. Histopathology 1995;27:205-218. Review.

7 Pietruszka M, Barnes L. Cystosarcoma phyllodes: a clinicopathologic analysis of 42 cases. Cancer 1978;41:1974-1983.

8 Birdsall SH, Summersgill BM, Egan M, et al. Additional copies of $1 \mathrm{q}$ in sequential samples from a phyllodes tumor of the breast. Cancer Genet Cytogenet 1995;83:111-114.

9 Birdsall SH, MacLennan KA, Gusterson BA. $t(6 ; 12)(q 23 ; q 13)$ and $t(10 ; 16)(q 22 ; p 11)$ in a phyllodes Tumor of Breast. Cancer Genet Cytogenet 1992;60: 74-77.

10 Dal Cin P, Moreman P, De Wever I, et al. Is i(1)(q10) a chromosome marker in phyllodes tumor of the breast? Cancer Genet Cytogenet 1995;83:174-175.

11 Dietrich CU, Pandis N, Teixeira MR, et al. Karyotypic changes in phyllodes tumors of the breast. Cancer Genet Cytogenet 1994;78:200-206.

12 Dietrich CU, Pandis N, Rizou H, et al. Cytogenetic findings in phyllodes tumors of the breast: karyotypic complexity differentiates between malignant and benign tumors. Hum Pathol 1997;78:1379-1382.

13 Ladesich J, Damjanov I, Persons D, et al. Complex karyotype in a low grade phyllodes tumor of the breast. Cancer Genet Cytogenet 2002;132:149-151.

14 Warso MA, Mehta RR, Hart GD, et al. A cell line derived from clinically benign phyllodes tumor: characterization and implications. Anticancer Res 1995;15:399-404.

15 du Manoir S, Speicher MR, Joos S, et al. Detection of complete and partial chromosome gains and losses by comparative genomic in situ hybridization. Hum Genet 1993;90:590-610.

16 Kallionemi A, Kallionemi OP, Sudar D, et al. Comparative genomic hybridization for molecular cytogenetic analysis of solid tumors. Science 1992;258: 818-821.

$17 \mathrm{Lu} \mathrm{YJ,} \mathrm{Birdsall} \mathrm{S,} \mathrm{Osin} \mathrm{P,} \mathrm{et} \mathrm{al.} \mathrm{PT} \mathrm{of} \mathrm{the} \mathrm{breast}$ analyzed by comparative genomic hybridization and association of increased 1q copy number with stromal overgrowth and recurrence. Genes Chromosomes Cancer 1997;20:275-281.

18 Bot FJ, Sleddens HF, Dinjens WN. Molecular assessment of clonality leads to the identification of a new germline TP53 mutation associated with malignant cystosarcoma phyllodes and soft tissue sarcoma. Diagn Mol Pathol 1998;7:295-301. 
19 Gatalica Z, Finkelsyein S, Lucio E, et al. P53 protein expression and gene mutation in phyllode tumor of the breast. Pathol Res Pract 2001;197:183-187.

20 Feakins RM, Mulcahy HE, Nickols CD, et al. p53 expression in phyllodes tumors is associated with histological features of malignancy but does not predict outcome. Histopathology 1999;35:162-169.

21 Sawyer EJ, Hanby AM, Rowan AJ, et al. The Wnt pathway, epithelial-stromal interactions, and malignant progression in phyllodes tumours. J Pathol 2002;196:437-444.

22 Sawyer EJ, Hanby AM, Poulsom R, et al. Beta-catenin abnormalities and associated insulin-like growth factor overexpression are important in phyllodes tumours and fibroadenomas of the breast. J Pathol 2003;5:627-632.

23 Carvalho S, e Silva AO, Milanezi F, et al. c-KIT and PDGFRA in breast phyllodes tumours: overexpression without mutations? J Clin Pathol 2004;57:1075-1079.

24 Sawyer EJ, Poulsom R, Hunt FT, et al. Malignant phyllodes tumours show stromal overexpression of c-myc and c-kit. J Pathol 2003;200:59-64.

25 Tse GM, Putti TC, Lui PC, et al. Increased c-kit (CD117) expression in malignant mammary PT. Mod Pathol 2004;17:827-831.

26 Kersting C, Kuijper A, Schmidt H, et al. Amplifications of the epidermal growth factor recePTor gene (egfr) are common in phyllodes tumors of the breast and are associated with tumor progression. Lab Invest 2006;86:54-61.

27 Mertens F, Johansson B, Hoglund M, et al. Chromosomal imbalance maps of malignant solid tumors: a cytogenetic survey of 3185 neoplasms. Cancer Res 1997;57:2765-2780.

28 Heim S, Mitelman F. Cancer Cytogenetics, 2nd edn. Wiley-Liss: New York, 1995, pp 369-388.

29 Mertens F, Johansson B, Mitelman F. Isochromosomes in neoplasia. Genes Chromosomes Cancer 1994;10: 221-230.

30 Ozaki T, Paulussen M, Poremba C, et al. Genetic imbalances revealed by comparative geneomic hybridization in Ewing tumors. Genes Chromosomes Cancer 2001;32:164-171.

31 Hislop RG, Pratt N, Stocks SC, et al. Karyotypic aberrations of chromosomes 16 and 17 are related to survival in patients with breast cancer. Br J Surg 2002;89:1581-1586.

32 Isola JJ, Kallionemi OP, Chu LW, et al. Genetic alterations detected by comparative genomic hybridization predict outcome in node-negative breast cancer. Am J Pathol 1995;147:905-911.

33 Muleris M, Almeida A, Gerbault-Sureau M, et al. Detection of DNA amplification in 17 primary breast carcinomas with homogeneously staining regions by a modified comparative genomic hybridization technique. Genes Chromosomes Cancer 1994;10:160-170.

34 Wei F, Ni J, Wu SS, et al. Cytogenetic studies of esophageal squamous cell carcinomas in the northern Chinese population by comparative genomic hybridization. Cancer Genet Cytogenet 2002;138:38-43.

35 Lillington DM, Kingston JE, Coen PG, et al. Comparative genomic hybridization of 49 primary retinoblasto- ma tumors identifies chromosomal regions associated with histopathology, progression, and patient outcome. Genes Chromosomes Cancer 2003;36:121-128.

36 Mairal A, Pinglier E, Gilbert E, et al. Detection of chromosome imbalances in retinoblastoma by parallel karyotype and CGH analyses. Genes Chromosomes Cancer 2000;28:370-379.

37 Hirai M, Yoshida S, Kashiwagi H, et al. 1q23 gain is associated with progressive neuroblastoma resistant to aggressive treatment. Genes Chromosomes Cancer 1999;25:261-269.

38 Knuutila S, Aalto Y, Autio K, et al. DNA copy number losses in human neoplasms. Am J Pathol 1999;155: 683-694.

39 Mitelman F, Johansson B, Mertens F (eds). Mitelman Database of Chromosome Aberrations in Cancer. http:// cgap.nci.nih.gov/chromosomes/Mitelman.

40 Chibon F, Mairal A, Freneaux P, et al. The RB1 gene is the target of chromosome 13 deletions in malignant fibrous histiocytoma. Cancer Res 2000;60:6339-6345.

41 Idbaih A, Coindre JM, Derre J, et al. Myxoid malignant fibrous histiocytoma and pleomorphic liposarcoma share very similar genomic imbalances. Lab Invest 2005;85:176-181.

42 Mairal A, Terrier P, Chibon F, et al. Loss of chromosome 13 is the most frequent genomic imbalance in malignant fibrous histiocytomas. A comparative genomic hybridization analysis of a series of 30 cases. Cancer Genet Cytogenet 1999;111:134-138.

43 Derre J, Lagace R, Nicolas A, et al. Leiomyosarcomas and most malignant fibrous histiocytomas share very similar comparative genomic hybridization imbalances: an analysis of a series of 27 leiomyosarcomas. Lab Invest 2001;81:211-215.

44 Lengauer C, Kinzler KW, Vogelstein B. Gentic instabilities in human cancers. Nature 1998;396:643-649.

45 Corzo C, Corominas JM, Tusquets I, et al. The MYC oncogene in breast cancer progression: from benign epithelium to invasive carcinoma. Cancer Genet Cytogenet 2006;165:151-156.

46 Ladanyi $\mathrm{M}$, Cha $\mathrm{C}$, Lewis $\mathrm{R}$, et al. MDM2 gene amplification in metastatic osteosarcoma. Cancer Res 1993;53:16-18.

47 Chibon F, Mariani O, Derre J, et al. A subgroup of malignant fibrous histiocytomas is associated with genetic changes similar to those of well-differentiated liposarcomas. Cancer Genet Cytogenet 2002;139: 24-29.

48 Pedeutour F, Suijkerbuijk RF, Forus A, et al. Complex composition and coamplification of SAS and MDM2 in ring and giant rod markers chromosomes in welldifferenciated liposarcoma. Genes Chromosomes Cancer 1994;10:85-94.

49 Reifenberger G, Ichimura $\mathrm{K}$, Reifenberger J, et al. Refined mapping of 12q13-q15 amplicons in human malignant gliomas suggests CDK4/SAS and MDM2 as independant amplification targets. Cancer Res 1996;56:5141-5145.

50 Jee KJ, Gong G, Ahn SH, et al. Gain in 1q is a common abnormality in phyllodes tumors of the breast. Anal Cell Pathol 2003;25:89-93. 